\title{
Postoperative enophthalmos correction and secondary midfacial reconstruction after complex orbital-maxillary fracture repair
}

\author{
Gordon S.K. Yau ${ }^{1}$, Aaron T. K. Chu ${ }^{1,2}$, Jacky W.Y. Lee ${ }^{1}$, Byron T.H. Chu' \\ Can Y.F. Yuen ${ }^{1}$ \\ ${ }^{1}$ Department of Ophthalmology, Caritas Medical Centre, Hong Kong Special \\ Administrative Region, People's Republic of China; ${ }^{2}$ Eye and Face Specialty Clinic, \\ Rm. 905A, 26 Nathan Road, Tsimshatsui, Hong Kong Special Administrative Region, \\ People's Republic of China
}

\begin{abstract}
Reconstruction of orbital wall and midfacial fractures pose a great challenge to oculoplastic surgeons as it may be associated with persistent postoperative enophthalmos, ocular misalignement, and facial asymmetry, requiring secondary reconstructive procedures. Cosmetically unacceptable postoperative enophthalmos should be revised. Several methods have been described including revision of the primary surgical implant or placement of additional implants. In the following case report, we describe a simple technique through a swinging eyelid approach to manage postoperative enophthalmos and secondary midfacial asymmetry following primary repair of a complex orbital-maxillary fracture.
\end{abstract}

Key words: orbital fracture; maxillary; enophthalmos; implant

\section{Introduction}

Complex orbital-maxillary fracture consists of fractures of the orbital wall and midfacial bones and it is the most pathology necessitating maxillofacial surgery. ${ }^{1}$ It is often associated with blunt midfacial trauma as a result of falls, road traffic accidents, or assault. ${ }^{2}$ Patients may present with diplopia, midfacial deformity, or enophthalmos. ${ }^{3}$ Reconstruction of these fractures is challenging because postoperative enophthalmos, ocular misalignment, and facial asymmetry may persist despite primary repair of the fracture and secondary reconstructive procedures are often required. ${ }^{4}$ Several surgical approaches have been previously described in the literature including revision of the primary surgical implant or placement of additional implants using nylon, titanium, bone graft. ${ }^{4,5}$ We report a case with persistent postoperative enophthalmos and facial asymmetry after orbital wall fracture repair, to illustrate a simple technique of using an enophthalmic implant and titanium mesh to correct for the cosmetic shortcomings.

Correspondence: Dr. Gordon S.K. Yau, Department of Ophthalmology, Caritas Medical Centre, 111 Wing Hong Street, Kowloon, Hong Kong Special Administrative Region, People's Republic of China. E-mail: skyau0303@gmail.com 


\section{Case report}

A 34-year-old woman with good past health was admitted for a right facial trauma after a slip and fall from the toilet without any loss of consciousness. Physical examination using the Hertel exophthalmometer revealed a right facial swelling and 2.0 $\mathrm{mm}$ enophthalmos ( $16 \mathrm{~mm}$ right eye, $19 \mathrm{~mm}$ left eye). Extra-ocular movement was full without diplopia. There was no afferent pupillary defect. Snellen visual acuity was 0.8 and 0.9 over the right and left eye respectively. Color vision test with Ishihara Pseudoisochromatic plates were normal in both eyes. Anterior segment examination and posterior segment examinations were normal. Non-contrast computed tomography (CT) of the orbit and face showed a right medial wall fracture, a large (>50\%) orbital floor fracture, and a maxillo-zygomatic complex fracture (Fig. 1a, b).
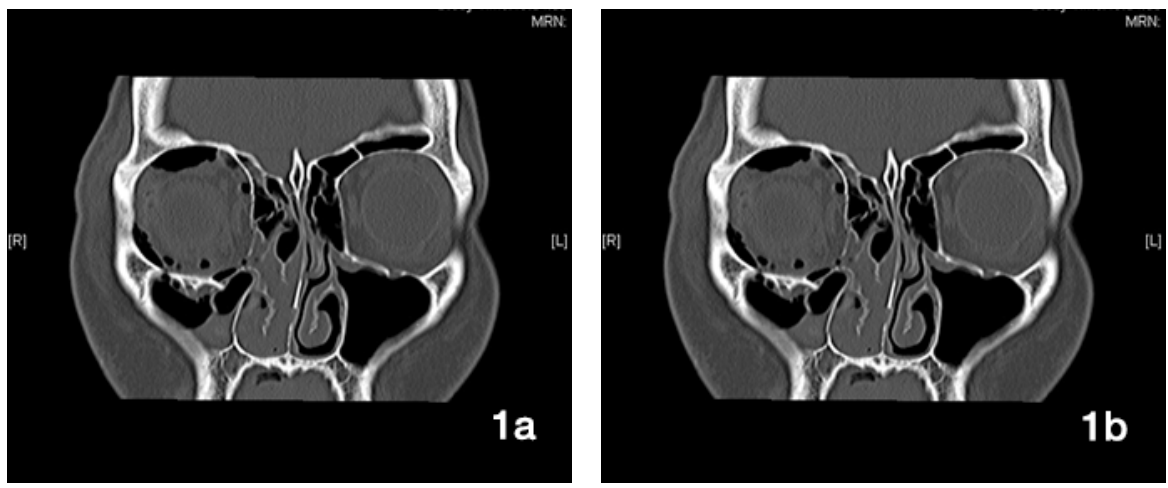

Fig. 1a, b. Computed tomography (CT) of the orbit and face.

Five days after the injury, right orbital fracture repair was performed under general anesthesia via a swinging eyelid approach with reconstruction using a 0.6-mm titanium mesh and screw fixation at the orbital rim. The maxillo-zygomatic

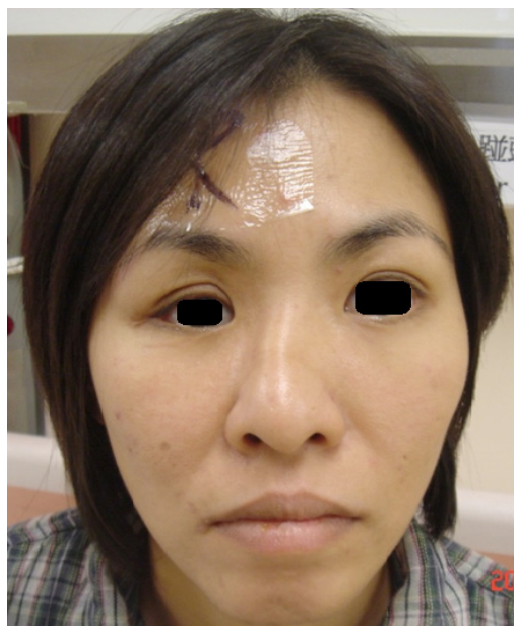
fracture was allowed to heal conservatively.

Three months postoperatively, there was a significant 4.0-mm right enophthalmos and facial asymmetry with loss of cheek prominence on the right (Fig. 2). CT orbit revealed an inadequate and imperfect restoration of orbital volume with significant hypoglobus and enophthalmos (Fig. 3).

The revision operation was performed under general anesthesia through a swinging eyelid approach to correct the residual enophthalmos and to reconstruct the midfacial asymmetry. Intra-operatively, the

Fig. 2. Three months postoperative showed right side enopthalmos, hypoglobus and facial asymmetry. 


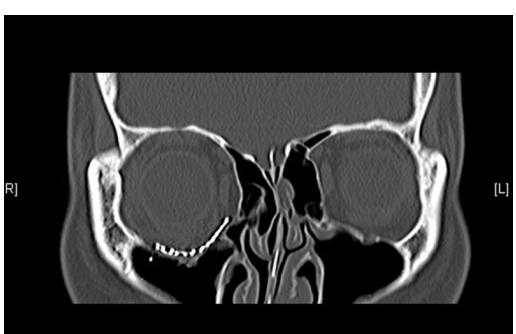

Fig. 3. CT Scan of orbit after primary repair.

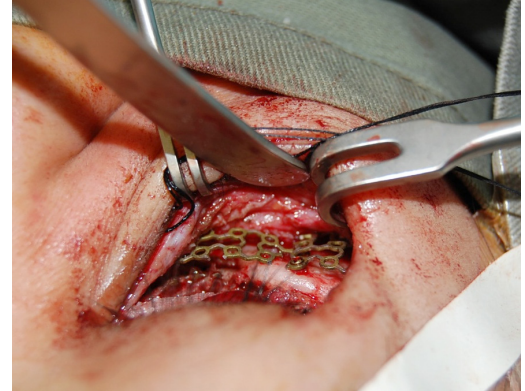

Fig. 4. Titanium mesh and plates to reconstructed the facial asymmetry.

titanium mesh was found to be densely adhered to scar tissue and could not be revised. Instead, orbital volume restoration was performed by the insertion of a high-density porous polyethylene (Medpor', Stryker, 2825 Airview Boulevard, Kalamazoo, Michigan 49002, USA) wedged enophthalmic implant (Large-Right: $28 \mathrm{~mm} \times 40 \mathrm{~mm} \times 7.5 \mathrm{~mm}$ ). To reconstruct the midface prominence, two titanium mesh and plates were fixed to the maxilla-zygomatic bone, configured along the facial contour (Fig. 4).

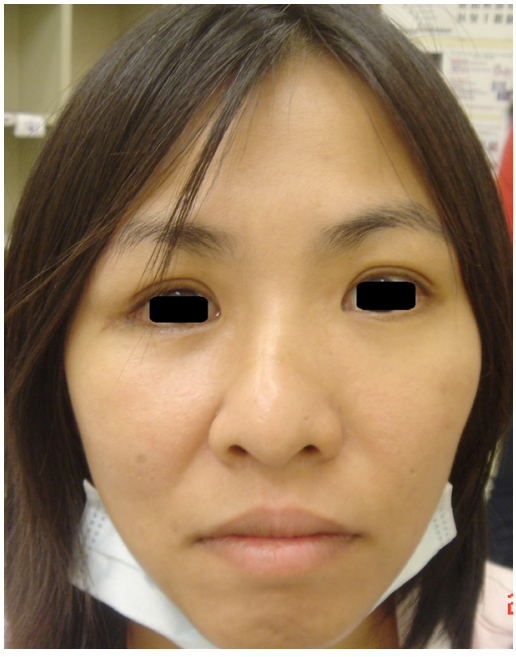

Four months postoperatively, the patient's enophthalmos significantly improved (1 $\mathrm{mm}$ right enopthalmos measured with the Hertel exophthalmeter) along with subjective cosmetic improvement (Fig. 5).Visual acuity of the right eye was static and there was no optic nerve dysfunction (visual acuity $=0.8$, no afferent papillary defect, normal color vision). No ocular motility problem was detected. A follow-up CT orbit revealed a much restored radiological configuration of the right orbit and face (Fig. 6 a, b).

Fig. 5. Postoperative clinical photo after revision surgery.

\section{Discussion}

Surgical repair of complex maxillary-orbital fractures is not uncommonly associated with postoperative complications. ${ }^{3}$ The frequency of postoperative enophthalmos following orbital wall fracture ranges from $3.7 \%$ to $7.0 \% .6,7$ The underlying cause of persistent enophthalmos is often inadequate orbital volume restoration, but some authors suggested that orbital fat atrophy may be the culprit. ${ }^{8}$ Cosmetically unacceptable enophthalmos should be revised. Methods include revision of the surgical implant or placement of additional enophthalmic implants. ${ }^{5}$ 

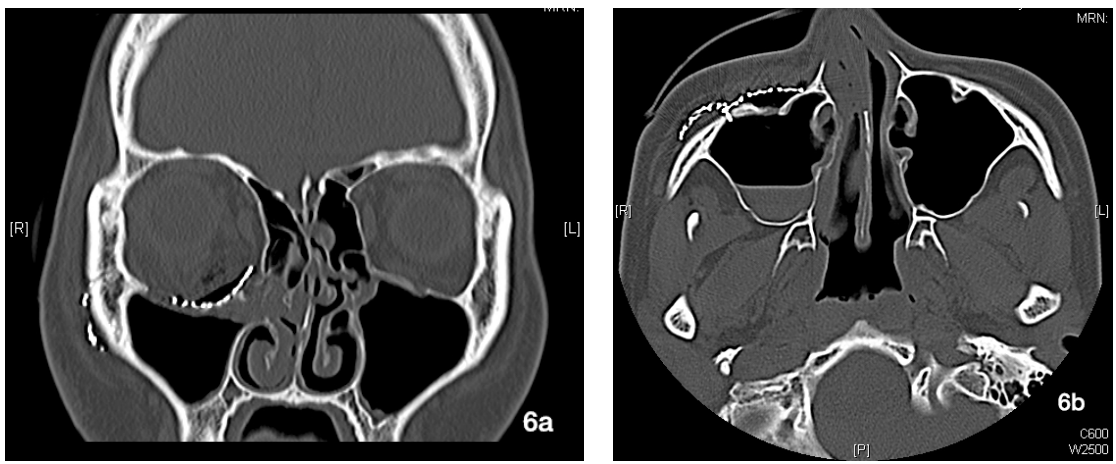

Fig. 6a,b. CT Scan of orbit and face after revisional surgery.

Lee et al. described a technique of orbital wall reconstruction of affected orbital wall combined with orbital decompression of the contralateral orbital wall to manage the residual enophthalmos. ${ }^{9}$

Osteotomy and four-point fixation techniques have been described in Lu et al's series in the late correction of midfacial fractures. ${ }^{10}$ Medpor enophthalmic implant was reported to be useful for augmenting the orbital volume and correcting the enophthalmos as well as hypoglobus in the primary repair of long-standing orbital wall fractures. ${ }^{11}$ Medpor enophthalmic implants mimics the contour of the orbital floor to restore both volume and shape. It is commercially available in two orientations (left and right) and two sizes (regular $-2 \mathrm{~mL}$ volume and large $-3 \mathrm{~mL}$ volume).

Previous studies have reported the use of Medpor enophthalmic implants for primary repair of late orbital fractures or the use of osteotomy and four-point bone fixation with screws for secondary revisions. This is one of the few reports in the literature of using only a Medpor enophthalmic implant and titanium meshes to secondarily restore orbital volume and correct midfacial asymmetry after primary orbital wall fracture repair.

The high-density porous polyethylene enophthalmic wedge implants have previously been described to correct post-enucleation socket syndrome. ${ }^{12,13}$ The additional volume to posterior orbit in a seeing eye may potentially compromise the optic nerve function or even cause ocular motility disorders. We report a case of postoperative enophthalmos managed successfully with Medpor wedge-implant with improvement in comesis and preservation of vision and optic nerve functions.

\section{Disclosures}

The authors declare no financial or proprietary interest. Informed patient consent was obtained prior to all surgical procedures. Consent was obtained for the release and publication of patient clinical and surgical photos and radiological investigations. 


\section{References}

1. Nardi P, Acocella A, Acocella G. Sequelae of zygomatic-orbito-maxillary fractures. Report of 70 cases and review of literature. Minerva Stomatol 2003;52(6):261-266.

2. al-Qurainy IA, Stassen LF, Dutton GN, Moos KF, el-Attar A. The characteristics of midfacial fractures and the association with ocular injury: a prospective study. $\mathrm{Br} J$ Oral Maxillofac Surg 1991;29(5):291-301.

3. al-Qurainy IA, Stassen LF, Dutton GN, Moos KF, el-Attar A. Diplopia following midfacial fractures. $\mathrm{Br}$ J Oral Maxillofac Surg 1991;29(5):302-307.

4. Iliff NT. The ophthalmic implications of the correction of late enophthalmos following severe midfacial trauma. Trans Am Ophthalmol Soc 1991;89:477-548.

5. Joseph JM and Glavas IP. Orbital fractures: a review. Clin Ophthalmol 2011;5: 95-100.

6. Gosau M, Schöneich M, Draenert FG, Ettl T, Driemel O, Reichert TE. Retrospective analysis of orbital floor fractures--complications, outcome, and review of literature. Clin Oral Investig 2011;15(3):305313. doi: 10.1007/s00784-010-0385-y. Epub 2010 Feb 18.

7. Banu M. Hossal and Randall L. Beatty. Diplopia and enophthalmos after surgical repair of blowout fracture. Orbit 2002;21(1):27-33. doi: 10.1076/orbi.21.1.27.2598.

8. Cole P, Kaufman Y, Hollier L. Principles of facial trauma: orbital fracture management. J Craniofac Surg 2009;20(1):101-104.

9. Lee JH, Park WY, Nam HJ, Kim YH. Correction of Persistent Enophthalmos after Surgical Repair of Blow Out Fracture Using Orbital Decompression Technique of Contralateral Eye .J Korean Cleft Palate-Craniofac Assoc 2008(2):101-104.

10. Lu W, Zhou H, Xiao C, Shen Q, Lin M, Fan X. Late correction of orbital-zygomatic-maxillary fractures combined with orbital wall fractures. J Craniofac Surg 2012;23(6):1672-1676. doi: 10.1097/ SCS.0b013e318266f963.

11. Kempster R, Beigi B,Galloway GD. Use of Enophthalmic Implants in the Repair of Orbital Floor Fractures. Orbit 2005;24(4):219-225. doi: 10.1080/01676830500182838.

12. Phan LT, Hwang TN, McCulley TJ. Evisceration in the modern age. Middle East Afr J Ophthalmol 2012;19:24-33.

13. Kim HE, Jang SY, Yoon JS. Combined orbital floor wedge implant and fornix reconstruction for post-enucleation sunken socket syndrome. Plast Reconstr Surg 2014; Feb 24. [Epub ahead of print]. 